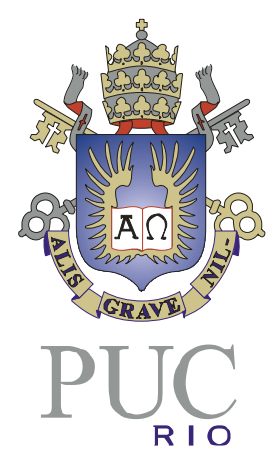

Manoel Roberto Aguirre de Almeida

\title{
Sistema Híbrido Neuro-Fuzzy-Genético para Mineração Automática de Dados
}

Dissertação de Mestrado

Dissertação apresentada como requisito parcial para obtenção do título de Mestre pelo Programa de PósGraduação em Engenharia Elétrica da PUC-Rio.

Orientadores:

Marley M. B. R. Velasco Marco Aurélio C. Pacheco 


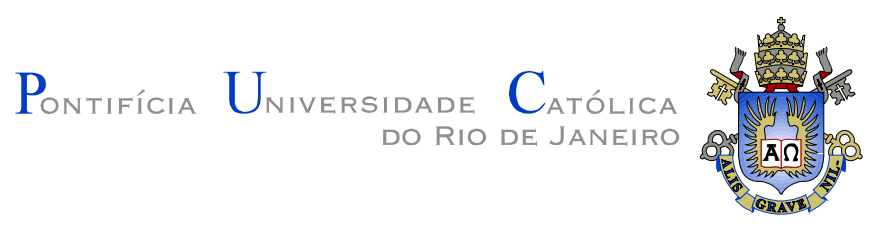

Manoel Roberto Aguirre de Almeida

\title{
Sistema Híbrido Neuro-Fuzzy-Genético para Mineração Automática de Dados
}

Dissertação apresentada como requisito parcial para obtenção do título de Mestre pelo Programa de Pós-Graduação em Engenharia Elétrica da PUC-Rio. Aprovada pela Comissão Examinadora abaixo assinada.

\author{
Prof. Marco Aurélio C. Pacheco \\ Orientador \\ Prof. Ricardo Tanscheit \\ PUC-Rio \\ Prof $^{\mathrm{a}}$. Karla T. Figueiredo Leite \\ PUC-Rio \\ Prof. Valmir C. Barbosa \\ UFRJ
}

Prof. Leandro dos Santos Coelho

PUC-PR

Prof. Flávio Joaquim de Souza UERJ

Prof. José Eugênio Leal Coordenador Setorial do Centro Técnico Científico - PUC-Rio

Rio de Janeiro, 25 de março de 2004 
Todos os direitos reservados. É proibida a reprodução total ou parcial do trabalho sem autorização da universidade, do autor e do orientador.

\section{Manoel Roberto Aguirre de Almeida}

Graduou-se em Engenharia de Computação pela PUCRio em Julho de 2001. Iniciou seus estudos de mestrado na área de Métodos de Apoio à Decisão no Departamento de Elétrica em Fevereiro de 2002.

Ficha Catalográfica

Almeida, Manoel Roberto Aguirre de

Sistema híbrido neuro-fuzzy-genético para mineração automática de dados / Manoel Roberto Aguirre de Almeida ; orientadores: Marley M. B. R. Velasco, Marco Aurélio C. Pacheco. - Rio de Janeiro : PUC, Departamento de Engenharia Elétrica, 2004.

112 f. : il. ; $30 \mathrm{~cm}$

Dissertação (mestrado) - Pontifícia Universidade Católica do Rio de Janeiro, Departamento de Engenharia Elétrica.

Incluí referências bibliográficas.

1. Engenharia elétrica - Teses. 2. Mineração de dados. 3. Sistemas neuro-fuzzy. 4. Coevolução genética. I. Velasco, Marley M. B. R. II. Pacheco, Marco Aurélio C. III. Pontifícia Universidade Católica do Rio de Janeiro. Departamento de Engenharia Elétrica. IV. Título. 


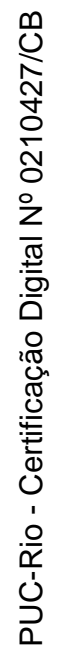

À minha família 


\section{Agradecimentos}

A Deus por ter me permitido existir e ter me guiado nesta jornada.

Ao CNPq, pelo apoio financeiro.

À Profa. Dra. Marley Maria B. R. Vellasco e ao Prof. Dr. Marco Aurélio C. Pacheco, orientadores desta tese, pelo apoio, carinho, incentivo e confiança depositados.

Ao meu pai por não medir esforços para me dar a melhor educação e me apoiar em todos os momentos do desenvolvimento deste trabalho.

À minha mãe que sempre sonhou em presenciar este momento e que, com certeza, em algum lugar está muito feliz por minha conquista.

Ao meu avô José e meus tios por acreditarem em mim e estarem sempre presentes com seu carinho.

À Raquel Andrade, pelo carinho, confiança, amor e, principalmente, pela paciência ao longo da elaboração desta dissertação.

Aos amigos João Machado e Danilo Tuler pela amizade e companheirismo nos momentos de sufoco.

Aos amigos do ICA com quem sempre pude contar e tirar dúvidas fundamentais no processo de pesquisa.

À Pontifícia Universidade Católica do Rio de Janeiro. 


\section{Resumo}

Manoel Roberto Aguirre de Almeida. Sistema Híbrido Neuro-FuzzyGenético para Mineração Automática de Dados. Rio de Janeiro, 2004. 112p. Dissertação de Mestrado - Departamento de Engenharia Elétrica, Pontifícia Universidade Católica do Rio de Janeiro.

Esta dissertação apresenta a proposta e o desenvolvimento de um sistema de mineração de dados inteiramente automático. O objetivo principal é criar um sistema que seja capaz de realizar a extração de informações obscuras a partir de bases de dados complexas, sem exigir a presença de um especialista técnico para configurá-lo. O sistema híbrido neuro-fuzzy hierárquico com particionamento binário (NFHB) vem apresentando excelentes resultados em tarefas de classificação de padrões e previsão, além de possuir importantes características não encontradas em outros sistemas similares, entre elas: aprendizado automático de sua estrutura; capacidade de receber um número maior de entradas abrangendo um maior número de aplicações; e geração de regras lingüísticas como produto de seu treinamento. Entretanto, este modelo ainda necessita de uma complexa parametrização inicial antes de seu treinamento, impedindo que o processo seja automático em sua totalidade. $O$ novo modelo proposto busca otimizar a parametrização do sistema NFHB utilizando a técnica de coevolução genética, criando assim um novo sistema de mineração de dados completamente automático. O trabalho foi realizado em quatro partes principais: avaliação de sistemas existentes utilizados na mineração de dados; estudo do sistema NFHB e a determinação de seus principais parâmetros; desenvolvimento do sistema híbrido neuro-fuzzy-genético automático para mineração de dados; e o estudo de casos.

No estudo dos sistemas existentes para mineração de dados buscou-se encontrar algum modelo que apresentasse bons resultados e ainda fosse passível de automatização. Várias técnicas foram estudadas, entre elas: Métodos Estatísticos, Árvores de Decisão, Associação de Regras, Algoritmos Genéticos, Redes Neurais Artificiais, Sistemas Fuzzy e Sistemas Neuro-Fuzzy. O sistema NFHB foi escolhido como sistema de inferência e extração de regras para a realização da mineração de dados. Deste modo, este modelo foi estudado e seus parâmetros mais importantes foram determinados. Além disso, técnicas de seleção de variáveis de entradas foram investigadas para servirem como opções para o modelo. Ao final, foi obtido um conjunto de parâmetros que deve ser automaticamente determinado para a completa configuração deste sistema. 
Um modelo coevolutivo genético hierárquico foi criado para realizar com excelência a tarefa de otimização do sistema NFHB. Desta forma, foi modelada uma arquitetura hierárquica de Algoritmos Genéticos (AG's), onde os mesmos realizam tarefas de otimização complementares. Nesta etapa, também foram determinados os melhores operadores genéticos, a parametrização dos AG's, a melhor representação dos cromossomas e as funções de avaliação. O melhor conjunto de parâmetros encontrado é utilizado na configuração do NFHB, tornando o processo inteiramente automático.

No estudo de casos, vários testes foram realizados em bases de dados reais e do tipo benchmark. Para problemas de previsão, foram utilizadas séries de carga de energia elétrica de seis empresas: Cerj, Copel, Eletropaulo, Cemig, Furnas e Light. $\mathrm{Na}$ área de classificação de padrões, foram utilizadas bases conhecidas de vários artigos da área como Glass Data, Wine Data, Bupa Liver Disorders e Pima Indian Diabetes. Após a realização dos testes, foi feita uma comparação com os resultados obtidos por vários algoritmos e pelo NFHB original, porém com parâmetros determinados por um especialista.

Os testes mostraram que o modelo criado obteve resultados bastante satisfatórios, pois foi possível, com um processo completamente automático, obter taxas de erro semelhantes às obtidas por um especialista, e em alguns casos taxas menores. Desta forma, um usuário do sistema, sem qualquer conhecimento técnico sobre os modelos utilizados, pode utilizá-lo para realizar mineração de bancos de dados, extraindo informações e até mesmo conhecimento que podem auxiliá-lo em processos de tomada de decisão, o qual é o objetivo final de um processo de Knowledge Data Discovery.

\section{Palavras-chave}

Mineração de Dados; Sistemas Neuro-Fuzzy; Coevolução Genética 


\section{Abstract}

Manoel Roberto Aguirre de Almeida. Hibrid Neuro-Fuzzy-Genetic System for Automatic Data Mining. Rio de Janeiro, 2004. 112p. MSc Dissertation - Departamento de Engenharia Elétrica, Pontifícia Universidade Católica do Rio de Janeiro.

This dissertation presents the proposal and the development of a totally automatic data mining system. The main objective is to create a system that is capable of extracting obscure information from complex databases, without demanding the presence of a technical specialist to configure it. The Hierarchical Neuro-Fuzzy Binary Space Partitioning model (NFHB) has produced excellent results in pattern classification and time series forecasting tasks. Additionally, it provides important features that are not present in other similar systems, such as: automatic learning of its structure; ability to deal with a larger number of input variables, thus increasing the range of possible applications; and generation of linguistic rules as a result of its training process. However, this model depends on a complex configuration process before the training is performed, hindering to achieve a totally automatic system. The model proposed in this Dissertation tries to optimize the NFHB system parameters by using the genetic coevolution technique, thus creating a new automatic data mining system. This work consisted of four main parts: evaluation of existing systems used in data mining; study of the NFHB system and definition of its main parameters; development of the automatic hybrid neuro-fuzzy-genetic system for data mining; and case studies.

In the study of existing data mining systems, the aim was to find a suitable model that could yield good results and still be automated. Several techniques have been studied, among them: Statistical methods, Decision Trees, Rules Association, Genetic Algorithms, Artificial Neural Networks, Fuzzy and NeuroFuzzy Systems. The NFHB System was chosen for inference and rule extraction in the data mining process. In this way, this model was carefully studied and its most important parameters were determined. Moreover, input variable selection techniques were investigated, to be used with the proposed model. Finally, a set of parameters was defined, which must be determined automatically for the complete system configuration.

A hierarchical coevolutive genetic model was created to execute the system optimization task with efficiency. Therefore, a hierarchical architecture of 
genetic algorithms (GAs) was created, where the GAs execute complementary optimization tasks. In this stage, the best genetic operators, the GAs configuration, the chromossomes representation, and evaluation functions were also determined. The best set of parameters found was used in the NFHB configuration, making the process entirely automatic.

In the case studies, various tests were performed with benchmark databases. For forecasting problems, six electric load series were used: Cerj, Copel, Eletropaulo, Cemig, Furnas and Light. In the pattern classification area, some well known databases were used, namely Glass Data, Wine Data, Bupa Liver Disorders and Pima Indian Diabetes. After the tests were carried out, a comparison was made with known models and with the original NFHB System, configured by a specialist.

The tests have demonstrated that the proposed model generates satisfactory results, producing, with an automatic process, similar errors to the ones obtained with a specialist configuration, and, in some cases, even better results can be obtained. Therefore, a user without any technical knowledge of the system, can use it to perform data mining, extracting information and knowledge that can help him/her in decision taking processes, which is the final objective of a Knowledge Data Discovery process.

\section{Keywords}

Data Mining; Neuro-Fuzzy Systems; Genetic Coevolution 


\section{Sumário}

1 Introdução 17

1.1. Motivação 17

1.2. Objetivos 18

1.3. Descrição do Trabalho 19

1.4. Organização da Tese 21

2 Sistemas de Mineração de Dados 22

2.1. Introdução 22

2.2. Classificação de Padrões 24

2.3. Previsão de Séries Temporais $\quad 24$

2.4. Técnicas de Mineração de Dados 25

2.4.1. Métodos Estatísticos 25

2.4.1.1. Discriminante Linear de Fisher 25

2.4.1.2. Classificadores Bayesianos 26

2.4.1.3. Redes Bayesianas 27

2.4.2. Árvores de Decisão $\quad 27$

2.4.2.1. ID3 e C4.5 28

2.4.2.2. CART (Classification And Regression Trees) 29

2.4.2.3. FID3.1 29

2.4.3. Regras de Associação $\quad 30$

2.4.4. Algoritmos Genéticos 30

2.4.4.1. GA-Miner 31

2.4.4.2. Rule Evolver 31

2.4.5. Redes Neurais Artificiais 31

2.4.6. Sistemas Neuro-Fuzzy 33

2.4.6.1. Sistema ANFIS 34

2.4.6.2. FSOM 36

$\begin{array}{ll}\text { 2.4.6.3. NEFCLASS } & 37\end{array}$

3 Sistema Neuro-Fuzzy Hierárquico BSP (NFHB) 39

3.1. Introdução 39 
3.2. O Particionamento BSP 40

3.3. A Célula Básica NFHB 41

3.4. A Arquitetura NFHB $\quad 45$

3.5. O Algoritmo de Aprendizado $\quad 47$

3.6. Técnicas para Atualização dos Pesos Fuzzy 52

3.6.1. Método de Gauss-Seidel 53

3.6.2. Método Resilient Back Propagation (RProp) 55

3.7. Estratégias para Seleção de Variáveis de Entrada 58

3.7.1. Método SIE $\quad 59$

3.7.2. Método LSE $\quad 61$

3.7.3. Método do Modelo Adaptado Anfis 61

3.8. Resumo dos Parâmetros do Sistema NFHB 62

4 Sistemas Coevolutivos $\quad 64$

4.1. Introdução $\quad 64$

4.2. O Modelo Coevolutivo Tradicional 65

4.3. Arquitetura Coevolutiva Hierárquica 68

5 Sistema Híbrido Neuro-Fuzzy-Genético para Mineração Automática de Dados72

5.1. Introdução

5.2. O Sistema Evolutivo de Otimização dos Parâmetros 72

5.2.1. Funções de Avaliação $\quad 75$

5.2.1.1. Previsão de Séries Temporais 76

5.2.1.2. Classificação de Padrões $\quad 78$

5.2.2. Configuração dos Algoritmos Genéticos 78

5.2.2.1. Previsão de Séries Temporais 82

6 Estudo de Casos $\quad 84$

6.1. Descrição

6.2. Previsão de Séries Temporais $\quad 85$

6.2.1. Séries de Carga Elétrica $\quad 85$

6.3. Classificação de Padrões 94

6.3.1. Base de Dados Glass Data 94

6.3.2. Base de Dados Wine Data 96

6.3.3. Base de Dados Pima Indians Diabetes $\quad 97$

6.3.4. Base de Dados Bupa Liver Disorders 98

6.3.5. Comentários sobre os Resultados dos Parâmetros 100 
6.4. Comparação com Resultados sem Otimização

7 Conclusões e Trabalhos Futuros

105

7.1. Conclusões

105

7.2. Trabalhos Futuros

106

8 Referências Bibliográficas

108

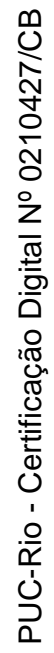




\section{Lista de figuras}

Figura 1: Fases do Processo de KDD 22

Figura 2: Exemplo de uma Árvore de Decisão 28

Figura 3: Exemplo de Topologia de Uma Rede Neural Artificial Perceptron

Multicamadas 32

Figura 4: Exemplo de Arquitetura de um Sistema ANFIS 35

Figura 5: Exemplo de Arquitetura de um Sistema FSOM 36

Figura 6: Exemplo de Arquitetura do Sistema NEFCLASS 38

Figura 7: Tipos Comuns de Particionamento dos Sistemas Neuro-Fuzzy 39

Figura 8: (a) Exemplo de Particionamento BSP; (b) Árvore do Particionamento

Figura 9: Esquema Simplificado de uma Célula Básica NFHB 42

Figura 10: Interior de uma Célula Básica NFHB 42

Figura 11: Formato das Funções de Pertinência da Célula NFHB 43

Figura 12: Célula NFHB na forma de uma Rede Neuro-Fuzzy 44

Figura 13: Exemplo de arquitetura NFHB 45

Figura 14: Particionamento Correspondente a Estrutura do Exemplo 46

Figura 15: Árvore de Particionamentos $\quad 46$

Figura 16: Algoritmo de Aprendizado do Sistema NFHB 48

Figura 17: Gráfico de Exemplo de Erros de Treinamento e Validação 50

Figura 18: Mecanismo de particionamento com uso de combinação linear das entradas 52

Figura 19: Exemplo de Arquitetura Coevolutiva Tradicional com 3 AG's 66

Figura 20: Diagrama de Seqüência de uma Arquitetura Coevolutiva Tradicional

Figura 21: Exemplo de Arquitetura Coevolutiva Hierárquica com 4 AG's e 3 Níveis

Figura 22: Diagrama de Seqüência de uma Arquitetura Coevolutiva Hierárquica

Figura 23: Hierarquia de Parâmetros do Sistema NFHB 73

Figura 24: Parâmetros do Sistema NFHB Agrupados 73

Figura 25: Diagrama de Atividade da Seleção de Indivíduos das Populações Inferiores 
Figura 26: Séries real e prevista da Copel 90

Figura 27: Séries real e prevista da Cemig $\quad 91$

Figura 28: Séries real e prevista da Light $\quad 91$

Figura 29: Séries real e prevista de Furnas $\quad 92$

Figura 30: Séries real e prevista da Cerj 93

Figura 31: Séries real e prevista da Eletropaulo 93

Figura 32: Exemplo de Codificação da Saída do NFHB para Classificação 94 


\section{Lista de tabelas}

Tabela 1: Resumo dos Parâmetros de Entrada do Sistema NFHB 63

Tabela 2: Tabela de Parâmetros de Configuração dos AGs 80

Tabela 3: Tabela de Valores dos Parâmetros de Configuração dos AG's 81

Tabela 4: Domínios dos Parâmetros do Sistema NFHB 82

Tabela 5: Tabela de Valores dos Parâmetros de Configuração do AG4 82

Tabela 6: Seqüências de entradas escolhidas pelos métodos Anfis, LSE e SIE para a série de Furnas $\quad 85$

Tabela 7: Seqüências de entradas escolhidas pelos métodos Anfis, LSE e SIE para a série da Light $\quad 86$

Tabela 8: Seqüências de entradas escolhidas pelos métodos Anfis, LSE e SIE para a série de Copel 86

Tabela 9: Seqüências de entradas escolhidas pelos métodos Anfis, LSE e SIE para a série de Eletropaulo $\quad 87$

Tabela 10: Seqüências de entradas escolhidas pelos métodos Anfis, LSE e SIE para a série da Cerj $\quad 87$

Tabela 11: Seqüências de entradas escolhidas pelos métodos Anfis, LSE e SIE para a série da Cemig $\quad 87$

Tabela 12: Tabela de parâmetros otimizados pelo Sistema Coevolutivo para todas as séries de carga. $\quad 88$

Tabela 13: Tabela de métricas de avaliação para todas as séries de carga analisadas

Tabela 14: Seqüências de entradas escolhidas pelos métodos Anfis, LSE e SIE para a base Glass Data $\quad 95$

Tabela 15: Parâmetros Otimizados para a base Glass Data 95

Tabela 16: Métricas do Sistema NFHB Otimizado para a base Glass Data 96

Tabela 17: Seqüências de entradas escolhidas pelos métodos Anfis, LSE e SIE para a base Wine Data 96

Tabela 18: Parâmetros Otimizados para a base Wine Data 97

Tabela 19: Métricas do Sistema NFHB Otimizado para a base Wine Data 97

Tabela 20: Seqüências de entradas escolhidas pelos métodos Anfis, LSE e SIE para a base Pima Indians Diabetes 98

Tabela 21: Parâmetros Otimizados para a base Pima Indians Diabetes 98 
Tabela 22: Métricas do Sistema NFHB Otimizado para a base Pima Indians Diabetes

Tabela 23: Seqüências de entradas escolhidas pelos métodos Anfis, LSE e SIE para a base Bupa Liver Disorders $\quad 99$

Tabela 24: Parâmetros Otimizados para a base Bupa Liver Disorders 99

Tabela 25: Métricas do Sistema NFHB Otimizado para a base Bupa Liver $\begin{array}{ll}\text { Disorders } & 100\end{array}$

Tabela 26: Tabela de parâmetros otimizados pelo Sistema Coevolutivo para todas as bases de classificação estudadas 100

Tabela 27: Tabela Comparativa entre o modelo NFHB configurado por especialista e o NFHB Otimizado por Coevolução em aplicações de previsão

101

Tabela 28: Tabela comparativa do Modelo Coevolutivo com outros métodos de previsão 102

Tabela 29: Tabela comparativa do Modelo Coevolutivo com outros métodos de previsão 102

Tabela 30: Tabela Comparativa entre o modelo NFHB configurado por especialista e o NFHB Otimizado por Coevolução em aplicações de classificação

103

Tabela 31: Tabela comparativa do Modelo Coevolutivo com outros métodos de Classificação 